\title{
SÍNDROME DEPRESSIVA: UM ESTUDO COM PACIENTES E FAMILIARES NO CONTEXTO DA DOENÇA RENAL CRÔNICA
}

Fabrycianne Gonçalves Costa

Universidade Federal da Paraíba

\author{
Maria da Penha de Lima Coutinho \\ Universidade Federal da Paraíba
}

\begin{abstract}
Resumo
No contexto da doença renal crônica, uma comorbidade frequente é a depressão, que pode estar relacionada aos fatores adversos do meio social ou inerentes ao próprio tratamento. Neste estudo, objetivou-se mensurar a sintomatologia depressiva em pacientes renais crônicos e seus cuidadores/familiares e relacioná-la as variáveis biossociodemográficas. Participaram do estudo 50 pacientes e 50 familiares, que responderam a um questionário sociodemográfico e à Escala Hospitalar de Ansiedade e Depressão. Constatou-se um índice de $20 \%$ de sintomatologia depressiva nos pacientes, sendo o índice maior entre os pacientes solteiros (50\%). Nos familiares, a prevalência dos sintomas depressivos foi de $46 \%$, observando-se correlação positiva entre o tempo de cuidado e a depressão. A incidência de depressão encontrada entre os familiares de pacientes suscita a necessidade de atendimentos psicossociais a esse grupo, que por vezes é tratado como sub-prioritário e nem sempre são assistidos pelas políticas públicas de saúde.
\end{abstract}

Palavras-chave: Depressão; insuficiência renal crônica; cuidadores familiares.

\section{DEPRESSIVE SYNDROME: A STUDY WITH PATIENTS AND FAMILIES IN THE CONTEXT OF CHRONIC RENAL DISEASE}

\begin{abstract}
In the context of chronic renal disease, one frequent comorbidity is depression, which is related to the adverse factors of the social environment or inherent to the treatment. In this research, we sought to measure depressive symptoms in chronic renal patients and their families, relating it to sociodemographic variables. Patients and their families answered a sociodemographic questionnaire and the Hospital Anxiety and Depression Scale (50 participants in each group). Almost $20 \%$ of the patients had depression, and the rate was greater among the single ones (50\%). In the family members, the prevalence of depressive symptoms was $46 \%$, and there was a positive correlation between the treatment duration and depression. The incidence of depression found among the patients' families draws attention for psychosocial care attentions directed to this group, which is often underprivileged and unassisted by public health policies.
\end{abstract}

Keywords: Depression; chronic renal failure; caregivers. 


\title{
SÍNDROME DEPRESIVA: UN ESTUDIO CON PACIENTES Y SUS FAMILIAS EN EL CONTEXTO DE LA ENFERMEDAD RENAL CRÓNICA
}

\begin{abstract}
Resumen
En el contexto de la enfermedad renal crónica, la depresión es una comorbilidad frecuente que puede estar relacionada con factores adversos del entorno social o inherentes al tratamiento. Este estudio tuvo como objetivo medir los síntomas depresivos en pacientes renales crónicos y sus cuidadores/familiares y relacionarlos con las variables sociodemográficas. El estudio incluyó a 50 pacientes y 50 miembros de la familia, que respondieron a un cuestionario sociodemográfico y la Escala Hospitalaria de Ansiedad y Depresión. Se encontró una tasa de $20 \%$ de la depresión en pacientes, la tasa más alta entre los pacientes individuales $(50 \%)$. En los miembros de la familia, la prevalencia de síntomas depresivos fue del $46 \%$, con una correlación positiva entre el tiempo de atención y depresión. La incidencia de la depresión que se encontró entre los familiares de los pacientes levanta la necesidad de atención psicosocial a este grupo, que a veces es tratado como sub-prioridad y no siempre son asistidos por las políticas de salud pública.
\end{abstract}

Palabras-clave: Depresión; insuficiencia renal crónica; cuidadores.

\section{INTRODUÇÃO}

A Doença Renal Crônica (DRC) é a fase mais avançada da doença renal, caracterizada pela perda progressiva e irreversível das múltiplas funções dos rins, que geram alterações nos diversos sistemas do organismo. Tal perda progressiva contribui para o fracasso da capacidade do corpo em manter os equilíbrios metabólicos e hidroeletrolíticos, resultando na retenção de ureia e outros resíduos nitrogenados no sangue (Brasil, 2014).

Por se tratar de uma doença assintomática, as pessoas desconhecem a sua existência até o seu diagnóstico. Na maioria das vezes, o diagnóstico só é realizado quando o quadro clínico se apresenta bastante avançado, necessitando imediatamente de tratamento para suprir a função renal (Brasil, 2014). Nesse caso, usualmente, a hemodiálise é o tratamento mais adotado para a substituição desta função. Caracteriza-se por ser um processo mecânico e extracorpóreo, que consiste na retirada de substâncias tóxicas e do excesso de líquido do organismo. Em geral, esse tratamento é realizado em hospitais, três vezes por semana, com duração média de quatro horas. Além destes cuidados, a pessoa deve seguir dietas e ingerir medicamentos (Rudnicki, 2014).

Sendo assim, o paciente é conduzido a conviver diariamente com uma doença incurável, que o obriga a uma forma de tratamento dolorosa, de longa duração e que provoca, juntamente com a evolução da doença e suas complicações, ainda maiores limitações e alterações de grande impacto. Essas limitações repercutem tanto em sua própria vida, quanto na do seu grupo familiar, afetando as várias dimensões do ser humano, sejam elas de ordem física, psicológica, econômica ou social (Ramos, Queiroz, \& Jorge, 2008).

Neste sentido, o paciente fica constantemente exposto a fatores adversos, inerentes à terapêutica da síndrome renal, como o tempo gasto nas sessões de hemodiálise, as constantes consultas médicas, os exames laboratoriais, as dietas 
e a expectativa de transplante, que, associados à frequente permanência em contextos hospitalares, contribuem para o surgimento da sintomatologia depressiva em tais pacientes e em seus cuidadores (Coutinho \& Costa, 2015; Ferreira \& Anes, 2010; Miyashita et al., 2009).

Estudos desenvolvidos por Kimmel et al. (2008) e Nifa e Rudnicki (2010) demonstram que a depressão parece ser a complicação psíquica mais frequente nos pacientes em tratamento de hemodiálise. Autores como Besier e Goldbeck (2011), Miyashita et al. (2009) e Maciel, Melo, Dias, Silva e Gouveia (2014) também constataram altos índices de depressão em cuidadores/familiares de pacientes de diversas ordens, relacionando-a as implicações do cuidar.

Segundo a Organização Mundial de Saúde - OMS (2016), o transtorno depressivo é caracterizado por alterações afetivas, cognitivas, volitivas e psicomotoras, que comprometem o funcionamento global do indivíduo, interferindo no seu cotidiano e nas suas relações sociais. Podendo indicar tanto um estado afetivo normal quanto um sintoma, uma síndrome, ou várias doenças (Ribeiro, Coutinho, \& Nascimento, 2010).

Estima-se que mais de 350 milhões de pessoas, em todo o mundo, sofram de depressão (OMS, 2016). No tocante aos pacientes em tratamento de hemodiálise, essa prevalência pode alcançar índices de até $100 \%$, dependendo dos instrumentos utilizados (Kimmel et al., 2008; Smith, Hong, \& Robson, 1985). Estudos mais recentes têm evidenciado prevalências menos abrangentes, a exemplo de Schuster, Feldens, Iser e Ghislandi (2015) e Costa, Coutinho, Melo e Oliveira (2014), que utilizaram o Inventário de Depressão de Beck (BDI) e constataram uma incidência de sintomas depressivos de $71 \%$ e $56,3 \%$ respectivamente.

Outros estudos assinalaram variações ainda menores, entre 10 e 30\% (Andrade, Sesso, \& Diniz, 2015; Kimmel et al., 2008). Stasiak, Bazan, Kuss, Schuinski, \& Baroni (2014) aplicaram tanto o BDI quanto a Escala Hospitalar de Ansiedade e Depressão (HADS) e verificaram maior prevalência de depressão com o BDI $(22,6 \%)$ e 9,3\% na HADS. Tal aspecto, pode ter ocorrido devido ao fato de o BDI conter questionamentos referentes a dados somáticos como alterações no peso e aumento ou diminuição do apetite, tendo em vista que esses aspectos são inconstantes na vida dessas pessoas e podem ser confundidos com a própria patologia renal. Entre os cuidadores, a sintomatologia depressiva também é frequente, podendo variar de $42 \%$ a $63 \%$ (Miyashita et al., 2009).

Os cuidadores informais são, normalmente, familiares, do sexo feminino, não remunerados, com pouco conhecimento técnico e pouco assistidos e orientados pelos servicos de saúde, que modificam suas rotinas para auxiliarem no cuidado com a saúde de seus ente-queridos (Marques, Landim, Collares, \& Mesquita, 2011). A família que cuida realiza tarefas de natureza diversificada, as quais estão relacionadas com as atividades da vida diária, abrangendo desde 
cuidados com a higiene, alimentação, tarefas domésticas, bem como, proteção, atenção, conforto, segurança, tratamento, repouso, socialização, administração da medicação, acompanhamento em consultas médicas, estendendo-se a outras atividades, como gerir aspectos econômicos (Cardoso, Vieira, Ricci, \& Mazza, 2012).

Em se tratando dos pacientes que necessitam da hemodiálise, os cuidadores também os acompanham durante as sessões. Além disso, existem os cuidados específicos com o cateter ou fístula, a administração de medicamentos de uso diário e contínuo e o controle da alimentação, pois esses pacientes têm a necessidade de seguir uma dieta rigorosa e a quantidade de líquidos a ser ingerida é mínima (Coutinho \& Costa, 2015; Gayomali et al., 2008). Quando esses pacientes são idosos, a atenção da família se estende para além da terapêutica específica referente à doença renal, abrangendo outras características que podem surgir devido à idade ou à própria patologia, como hipertensão, dificuldades de circulação, problemas cardíacos, diabetes, deficiência visual, incapacidade de andar, além de transtornos de humor ou cognitivos (Gayomali et al., 2008; Ribeiro, Marques, \& Ribeiro, 2009).

Deste modo, o cuidador sem suporte pode ser um futuro paciente com depressão, isto porque, como foi relatado por Marques et al. (2011), o familiar cuidador volta toda sua atenção para as necessidades imediatas do doente e deixa de cuidar de si. Dessa forma, o cuidar do paciente renal pode conduzir o familiar ao comprometimento de sua rotina, Ihes ocasionando diminuição das atividades sociais, alterações na saúde, problemas no trabalho, mudanças na vida familiar, entre outros. Sendo assim, tais compromentimentos podem acarretar diminuição tanto na qualidade de vida do cuidador como afetar o cuidado com o doente renal crônico (Marques et al., 2011).

Conforme Dias (2011), existe inter-relação entre o sofrimento do familiar cuidador e o do paciente, com consequências desfavoráveis para ambos, dessa forma, se a família é afetada pela depressão, as reações familiares também afetam o paciente. Percebe-se, portanto, que o processo de cuidar é complexo, influenciando e sendo influenciado pelo adoecer.

Diante do exposto, verifica-se a relevância de mensurar a sintomatologia depressiva em pacientes renais crônicos e seus familiares/cuidadores e relacioná-la as variáveis sociodemográficas. Desse modo, espera-se identificar a prevalência desta síndrome e compará-la entre os dois grupos de participantes, bem como, com as variáveis sociodemográficas (sexo, idade, estado civil, entre outras). Almeja-se que os resultados evidenciados nesse estudo, possam fornecer subsídios científicos para o desenvolvimento de políticas públicas, que auxiliem a atuação dos profissionais da saúde direcionadas para a prevenção e/ou tratamento da depressão nos pacientes e cuidadores de doentes renais crônicos. 


\section{MÉTODO}

Tratou-se de uma pesquisa descritiva, com uma abordagem quantitativa, realizada em três instituições hospitalares que disponibilizam o tratamento da hemodiálise para adultos e mantêm convênio com o Sistema Único de Saúde (SUS), localizadas no município de João Pessoa/PB.

\section{Participantes}

A amostragem foi do tipo não probabilístico e de conveniência. A pesquisa desenvolveu-se com dois grupos. O primeiro foi composto por 50 pacientes com DRC que atenderam aos seguintes critérios de inclusão: ser maior de 18 anos e estar em tratamento de hemodiálise por no mínimo três meses, pois considerouse que esse tempo seria um período de adaptação do paciente à hemodiálise. 0 segundo grupo foi formado por 50 cuidadores, todos familiares dos participantes do primeiro grupo, os quais foram definidos, no presente estudo, como aqueles que estavam acompanhando os pacientes à hemodiálise e os auxiliavam nos cuidados diários.

\section{Instrumentos}

Para a obtenção dos dados utilizou-se um questionário sociodemográfico, aplicado com o intuito de obter informações, tais como: sexo, idade, grau de escolaridade, situação conjugal, profissão e religião. Além disso, para os pacientes, questionou-se o tempo de diagnóstico e de hemodiálise. $E$, especificamente para os familiares, o tempo de acompanhamento e auxílio nos cuidados diários, bem como o grau de parentesco.

Aplicou-se ainda a Escala Hospitalar de Ansiedade e Depressão (HADS), instrumento adaptado à população brasileira por Botega, Bio, Zomignani, Garcia e Pereira (1995). Essa escala de rastreamento possui 14 itens, sete voltados para a avaliação da ansiedade (HADS-A) e sete para a depressão (HADS-D), cada item podendo ser pontuado de zero a três, compondo uma pontuação máxima de 21 pontos em cada componente avaliado. No presente estudo, foram utilizados exclusivamente os itens referentes a sub escala (HADS-D), na qual os níveis de sintomatologia depressiva foram categorizados da seguinte forma: sem sintomas depressivos (0-07), depressão leve (08-10), depressão moderada (1114) e depressão grave (15-21) (Botega et al., 1995; Marcolino, Mathias, Piccinini Filho, Guaratini, Suzuki, \& Alli, 2007).

No que se refere a consistência interna das sub escalas de ansiedade e depressão, os autores Botega et al. (1995) verificaram coeficientes de alfa de Cronbach, de 0,68 e 0,77, respectivamente. Entretanto, Marcolino et al. (2007) constataram maiores índices, respectivamente nas subescalas de ansiedade e depressão, tanto para pacientes internados $(0,84$ e 0,83$)$, quanto para população em geral $(0,84$ e 0,79$)$. Para avaliar a validade de critério e da confiabilidade com pacientes no pré-operatório, comparando com as escalas de Beck de 
ansiedade e depressão, constatou-se que a correlação da HADS, variou de 0,60 a 0,70 , sendo considerada uma correlação de média a forte, apresentando índices de consistência interna recomendáveis para instrumentos de triagem (Marcolino et al., 2007).

\section{Procedimentos}

Este estudo foi realizado considerando as condições éticas estabelecidas pela Comissão do Conselho Nacional de Saúde, pertinentes a pesquisas envolvendo seres humanos, de acordo com a Resolução 466/2012, e com constituição designada pela Resolução 246/97, sendo aprovado pelo Comitê de Ética em Pesquisa do Centro de Ciências da Saúde, da Universidade Federal da Paraíba, sob o protocolo de $n^{0} 392 / 11$.

Quanto ao procedimento de coleta de dados, os pacientes e familiares/cuidadores foram abordados na sala de espera para a hemodiálise. Inicialmente, cada participante assinou um Termo de Consentimento Livre e Esclarecido, e, na sequência, procedeu-se administração dos instrumentos, obedecendo-se à seguinte ordem: primeiro, o questionário sociodemográfico, em seguida a HADS-D. Os instrumentos foram aplicados individualmente e oralmente, lidos pelo pesquisador, devido à incapacidade de alguns participantes em responder por escrito. Destaca-se que as respostas dos pacientes não ocorreram na presença dos familiares. O tempo médio de aplicação dos instrumentos foi de 30 minutos.

Para a análise dos dados, utilizou-se o Pacote Estatístico para as Ciências Sociais (SPSS) para Windows versão 19.0. Foi empregada a estatística descritiva (média, desvio padrão, amplitude e frequências) para descrever o perfil da amostra estudada, a partir das variáveis do questionário sociodemográfico e para mensurar a prevalência dos sintomas depressivos. A estatística inferencial foi utilizada para verificar a existência de diferenças significativas no nível de sintomatologia depressiva entre familiares e pacientes. Para tanto, efetuou-se o teste $U$ de Mann-Whitney para amostras independentes, após ser constatada a não normalidade da distribuição da variável depressão por meio do teste Kolmogorov-Smirnov $(D(33)=0,117 ; \mathrm{p}=0,002)$.

Além destes, utilizou-se o qui-quadrado para amostras independentes e a correlação de Spearman. O primeiro, foi utilizado para testar a associação entre a sintomatologia depressiva e as variáveis (sexo, estado civil, ocupação, religião, ter outro familiar com a DRC e grau de parentesco) e o segundo, a relação entre esta sintomatologia e as variáveis (escolaridade, tempo de diagnóstico e tempo de tratamento). Destaca-se, que esses testes não-paramétricos foram utilizados porque as variáveis foram consideradas como categóricas.

Fez-se uso também do teste paramétrico, a correlação $r$ de Pearson para verificar a relação entre a sintomatologia depressiva com as variáveis (idade e 
tempo de acompanhamento/cuidados). Para este estudo foi adotado o nível de significância de $5 \%$, considerando $p \leq 0,05$.

\section{RESULTADOS E DISCUSSÃO}

Os resultados dos dados sociodemográficos dos participantes estão dispostos nas tabelas 1 e 2 . Verificou-se, que as idades dos pacientes variaram entre 20 e 73 anos $(M=45,0 ; D P=13,4)$ e todos realizavam três sessões de hemodiálise por semana. Na Tabela 1 , observa-se que a maioria dos pacientes era do sexo feminino representando $52 \%$, sendo $66 \%$ destes casados, $56 \%$ com escolaridade até o ensino fundamental. Quanto à religião, notou-se que $58 \%$ dos pacientes eram católicos. Em relação à ocupação, $88 \%$ responderam que eram aposentados. Notou-se ainda, que a maioria dos participantes tinha o diagnóstico da doença renal entre 1 a 3 anos $(M=2,54 ; D P=1,11)$ e apresentavam este mesmo tempo de hemodiálise $(M=2,18 ; \mathrm{DP}=1,04)$.

Tabela 1. Frequência e porcentagem (\%) das variáveis sociodemográficas dos pacientes com DRC $(n=50)$ 


\begin{tabular}{|c|c|c|}
\hline Variáveis & Frequência & $\%$ \\
\hline \multicolumn{3}{|l|}{ Sexo } \\
\hline Masculino & 24 & 48,0 \\
\hline Feminino & 26 & 52,0 \\
\hline \multicolumn{3}{|l|}{ Estado civil } \\
\hline Casado & 33 & 66,0 \\
\hline Solteiro & 17 & 34,0 \\
\hline \multicolumn{3}{|l|}{ Escolaridade } \\
\hline Analf./Fundamental & 28 & 56,0 \\
\hline Médio & 15 & 30,0 \\
\hline Superior & 07 & 14,0 \\
\hline \multicolumn{3}{|l|}{ Ocupação } \\
\hline Aposentados & 44 & 88,0 \\
\hline Outras & 06 & 12,0 \\
\hline \multicolumn{3}{|l|}{ Religião } \\
\hline Católica & 29 & 58,0 \\
\hline Evangélica & 15 & 30,0 \\
\hline Outras & 06 & 12,0 \\
\hline \multicolumn{3}{|l|}{ Tempo de diagnóstico } \\
\hline Menos de 1 ano & 10 & 20,0 \\
\hline De 1 a 3 anos & 17 & 34,0 \\
\hline De 4 a 6 anos & 09 & 18,0 \\
\hline Maior igual a 7 anos & 14 & 28,0 \\
\hline \multicolumn{3}{|l|}{ Tempo de hemodiálise } \\
\hline Menos de 1 ano & 15 & 30,0 \\
\hline De 1 a 3 anos & 19 & 38,0 \\
\hline De 4 a 6 anos & 08 & 16,0 \\
\hline Maior igual a 7 anos & 08 & 16,0 \\
\hline
\end{tabular}

Na Tabela 2 estão apresentados os resultados referentes aos cuidadores. Constatou-se que, dos 50 (cinquenta) participantes que constituíram a amostra, $82 \%$ eram do sexo feminino, com idades entre 18 a 68 anos $(M=40,7$; DP= $14,0)$ e, a maioria (70\%) era casada. Observou-se também, que $46 \%$ dos participantes tinham o ensino fundamental como maior nível de escolaridade e $40 \%$ afirmaram serem donas de casa. Por fim, constatou-se que a maior parte dos cuidadores acompanhavam seus familiares entre 1 e 3 anos $(M=1,66$; DP= $0,87)$ e que destes, $72 \%$ eram esposas e filhos. Esses resultados estão em congruência com um estudo de revisão realizado por Cardoso et al. (2012), os quais constataram a presença feminina no papel de cuidador principal, sejam elas, filhas ou esposas, casadas, de meia idade e com filhos, que predominantemente cuidam de seus progenitores ou cônjuges.

Essa predominância justifica-se pelo papel sociocultural que é imposto a figura feminina, como principal provedora dos cuidados à família e aos necessitados. Esta atividade, na maioria das vezes, é desempenhada com dedicação integral, derivada de uma construção social ideologicamente 
determinada e aceita, constituindo-se como uma verdade em uma obrigação moral (Cardoso et al., 2012).

Possivelmente, um outro desdobramento deve-se ao fato que essas cuidadoras a médio e longo prazo fiquem mais susceptíveis a adquirirem a síndrome da depressão, conforme ratificação da literatura, a qual aponta que as mulheres são mais afetadas que os homens (OMS, 2016).

Tabela 2. Frequência e porcentagem (\%) das variáveis sociodemográficas dos familiares/cuidadores $(n=50)$

\begin{tabular}{|c|c|c|}
\hline Variáveis & Frequência & $\%$ \\
\hline \multicolumn{3}{|l|}{ Sexo } \\
\hline Masculino & 09 & 82,0 \\
\hline Feminino & 41 & 18,0 \\
\hline \multicolumn{3}{|l|}{ Estado civil } \\
\hline Casado & 35 & 70,0 \\
\hline Solteiro & 15 & 30,0 \\
\hline \multicolumn{3}{|l|}{ Escolaridade } \\
\hline Analf./Fundamental & 23 & 46,0 \\
\hline Médio & 14 & 28,0 \\
\hline Superior & 13 & 26,0 \\
\hline \multicolumn{3}{|l|}{ Ocupação } \\
\hline Dona de casa & 20 & 40,0 \\
\hline Agricultor & 08 & 16,0 \\
\hline Estudante & 05 & 10,0 \\
\hline Outras & 17 & 34,0 \\
\hline \multicolumn{3}{|l|}{ Religião } \\
\hline Católica & 32 & 64,0 \\
\hline Evangélica & 16 & 32,0 \\
\hline Outras & 02 & 04,0 \\
\hline \multicolumn{3}{|c|}{$\begin{array}{l}\text { Tempo de acompanhamento/ } \\
\text { Cuidados }\end{array}$} \\
\hline Menos de 1 ano & 15 & 30,0 \\
\hline De 1 a 3 anos & 19 & 38,0 \\
\hline De 4 a 6 anos & 08 & 16,0 \\
\hline Maior igual a 7 anos & 08 & 16,0 \\
\hline \multicolumn{3}{|l|}{ Proximidade } \\
\hline Esposa & 20 & 40,0 \\
\hline Filhos & 16 & 32,0 \\
\hline Outros & 14 & 28,0 \\
\hline
\end{tabular}

Em relação aos resultados constatados por meio da HADS-D aplicada com o objetivo de mensurar a prevalência da sintomatologia depressiva, observou-se que na amostra de pacientes a pontuação variou de 0 a 18 pontos, com escore médio de 5,36 ( $\mathrm{DP}=3,49)$. A prevalência de depressão encontrada entre esses participantes foi de $20 \%$, e, desse montante, $12 \%$ possuíam sintomatologia 
depressiva leve, 6\% moderada e 2\% grave. Este nível de incidência está em consonância com a literatura, cujos índices se situam entre 10 e 100\% (Costa et al., 2014; Garcia et al., 2010; Kimmel et al., 2008; Smith et al., 1985).

Diante desses resultados, é possível inferir que os pacientes com DRC em tratamento de hemodiálise são mais suscetíveis em relação à população em geral, de serem acometidos pela sintomatologia da depressão, uma vez que a literatura preconiza que esta síndrome é frequente nessas pessoas, conforme dados da OMS (2016), aproximadamente $5 \%$ da população mundial sofrem de depressão. Nesses pacientes, a sintomatologia depressiva parece estar relacionada a mudanças na qualidade de vida, diminuição da imunidade e da capacidade funcional, relaxamento dos cuidados pessoais e adesão aos tratamentos e dietas (Cuker et al., 2010). Consequentemente, esses problemas acarretam o aumento do número de consultas ambulatoriais, internações e até mortalidade (Chilcot et al., 2010).

Por outro lado, o impacto do diagnóstico da DRC e a tomada de consciência do tratamento da hemodiálise, predispõe o paciente a outros tipos de enfermidades principalmente aquelas relacionadas as doenças psicoafetivas. Provocando, sentimentos de medo, tristeza, desespero e sofrimento ante a possibilidade da morte (Coutinho \& Costa, 2015).

Face a essas premissas torna-se imprescindível maior precaução no cuidado da atenção, promoção e prevenção da DRC, com a finalidade de otimizar os programas de políticas públicas voltados para a conscientização da comunidade, em geral, no que concerne aos fatores de risco que levam ao desencadeamento dessa doença, tais como: fatores genéticos, hipertensão arterial, diabetes, obesidade, proteinúria, tabagismo, entre outros, com o intuito de minimizar a morbimortalidade dessa população.

Quanto aos cuidadores, constatou-se uma pontuação mínima de 0 e máxima de 17 pontos $(M=6,84 ; \mathrm{DP}=3,76)$, observando-se um total de $46 \%$ de familiares/cuidadores com a sintomatologia depressiva, desses 30\% tinham sintomas leves de depressão, $10 \%$ moderados e $6 \%$ graves. A prevalência da depressão encontrada nos cuidadores deste estudo foi semelhante à constatada em outros estudos com cuidadores de pacientes crônicos de outras doenças, tais como demências (Miyashita et al., 2009; Sansoni et al., 2004).

Destaca-se, que a alta incidência da depressão encontrada nos cuidadores/familiares das pessoas com DRC, provavelmente pode estar diretamente relacionada aos cuidados proporcionados aos doentes renais crônicos, compreendendo desde os mais abrangentes, como as atividades da vida diária, até os específicos da patologia renal, como as restrições alimentares, administração da medicação, acompanhamento em consultas médicas e sessões de hemodiálise, entre outras (Vieira et al., 2011; Fernandes, 2009; Gayomali et al., 2008). Esses achados, provavelmente, podem indicar que a presença da 
depressão no cuidador alerta sobre o desgaste que o envolvimento afetivo e a sobrecarga de atividades têm em modificar a saúde mental dessas pessoas.

$\mathrm{Na}$ sequência, com o intuito de verificar se havia diferença significativa entre a sintomatologia depressiva dos cuidadores e pacientes, efetuou-se um teste $U$ de Mann-Whitney, obtendo resultados de 927 ( $z=-2,23)$, com probabilidade associada de 0,02, conforme as estatísticas descritivas, estas apontaram um maior nível de depressão para os cuidadores (mediana =7) comparada aos pacientes (mediana $=5$ ). Destaca-se, que o tamanho do efeito encontrado foi de $(r=0,22)$, apesar de ser considerado pequeno, este foi significativo (Fritz, Morris, \& Richler, 2012).

Esses resultados corroboram com os estudos desenvolvidos por Coutinho e Costa (2015), Marques et al. (2011) e Ribeiro et al. (2009) ao afirmarem que apesar dos cuidadores não estarem acometidos pela patologia renal, esta faz com que eles experienciam mudanças em seus estilos de vida, como, por exemplo, nos hábitos alimentares, limitações em suas vidas laborais, pois muitas vezes esses cuidadores abdicam do trabalho, ou tentam associá-las para acompanhar seus familiares nas sessões de hemodiálise e no cuidar cotidiano. Esta realidade, por suposto, impõe mudanças nos padrões de comportamento, podendo restringir também as atividades de lazer, desencadeando uma situação de sobrecaga psicológica.

Neste cenário, o impacto de conviver com uma pessoa doente implica em condição análoga de sofrimento, apesar dos cuidadores não estarem doentes fisiologicamente, a realidade da doença renal e sua terapêutica provocam limitações na vida dos pacientes e consequentemente para a vida dos cuidadores. As preocupações dos pacientes estão voltadas para a doença; já os familiares, estão envolvidos desde a própria doença, ao cuidar do ser doente (Costa, 2013). Dessa forma, é importante salientar que as doenças crônicas podem desencadear desequilíbrios psicoemocionais não apenas nos pacientes, mas também nas pessoas que estão perto deles, como é o caso de seus cuidadores (Sezer et al., 2003).

Contrariando os dados encontrados neste estudo, Saeed et al. (2012), ao medir e comparar a prevalência da depressão em pacientes renais e em seus cuidadores, utilizando o BDI, constataram que $75 \%$ dos pacientes e $33,4 \%$ dos cuidadores apresentaram sintomas depressivos de moderado à grave. Por outro lado, Asti et al. (2006) constataram similaridades entre as frequências da depressão em pacientes (10\%) e em seus cuidadores (9\%).

Arechabala, Catoni, Palma e Barrios (2011) em estudo realizado no Chile, por meio da escala de depresión del Centro de Estudios Epidemiológicos, observaram semelhanças na presença de depressão dos pacientes renais $(40,74 \%)$ e de seus cuidadores principais (43,82\%). Esses autores consideram que a carga do cuidado de um parente paciente implica em outras complicações, que surgem por estes negligenciarem de algum modo a sua saúde, por 
diminuírem as atividades sociais, aumentarem a carga de trabalho, terem restrições ou consequências econômicas, problemas sexuais e períodos prolongados de tempo dedicados aos pacientes, o que pode resultar em consequências na saúde dos cuidadores, ocasionando-lhes problemas como a depressão.

Para identificar a relação da sintomatologia depressiva com as variáveis sociodemográficas dos pacientes, utilizou-se a variável depressão como categórica ("presença ou ausência" de sintomatologia depressiva), sendo assim, foram realizados testes qui-quadrado para amostras independentes com as variáveis: sexo, ocupação, religião, ter outro familiar com a DRC e estado civil. Não foram constatadas associações significativas para as variáveis a seguir: sexo $\chi^{2}(1,100)=0,32, \mathrm{p}=0,41$; ocupação $\chi^{2}(1,100)=0,47, \mathrm{p}=0,65$; religião $\chi^{2}(2$, $100)=2,96, \mathrm{p}=0,22$; ter outro familiar com a $\operatorname{DRC} \chi^{2}(1,100)=1,27, \mathrm{p}=$ 0,22 .

Quanto ao estado civil dos pacientes, constatou-se significância marginal entre a associação das variáveis casado ou solteiro, com a presença ou ausência da sintomatologia depressiva $\chi^{2}(1,100)=3,76, p=0,06$. A partir do cruzamento destas variáveis, se pôde observar que existiram menos casos de depressão entre os casados, tanto em termos absolutos quanto em termos relativos, uma vez que mais de $50 \%$ dos solteiros apresentaram depressão enquanto que entre os casados, apenas $12 \%$ apresentaram depressão.

Nesse sentido, implica-se que para os pacientes estar casado parece ser um fator protecional para a depressão, já que a minoria destes (12\%) apresentou esta síndrome psicoafetiva. Moura Junior et al. (2008) corroboram com esses dados, ao estudarem a prevalência de transtornos psiquiátricos (incluindo a depressão) em pacientes renais, destacando que os pacientes solteiros apresentaram mais chance de diagnóstico de transtornos psiquiátricos, quando em comparação com os casados. Por sua vez, os viúvos e os separados tinham, respectivamente, de três a cinco vezes mais chances de transtornos, quando comparados aos casados.

Com a finalidade de verificar se existia associação entre as demais variáveis (escolaridade, tempo de diagnóstico e tratamento), utilizou-se a correlação de Spearman. Os resultados não apontaram significância estatística, entre a escolaridade e os índices de sintomas depressivos $\rho(1)=0,11, p=0,41$, entre o somatório da HADS-D e o somatório do tempo de diagnóstico da doença $\rho(1)=-0,70, p=0,63$, assim como, para o somatório do tempo de tratamento em hemodiálise $\rho(1)=0,03, \mathrm{p}=0,81$ ).

Em relação aos cuidadores/familiares, para identificar a relação das variáveis sociodemográficas com os sintomas da depressão, inicialmente, utilizou-se a correlação $r$ de Pearson para o somatório do tempo que os cuidadores acompanham seus familiares e o somatório da HADS-D. Verificou-se uma correlação significativa positiva e de qualidade moderada $r(1)=0,37, \mathrm{p}=$ 
0,008), sugerindo que quanto maior o tempo de cuidado, maior o nível de depressão desses acompanhantes.

O prolongamento do tempo de acompanhamento e cuidados dispensados, realizados por esses familiares pode desencadear a depressão, devido ao maior tempo de exposição às situações estressantes, como é o caso de estarem disponíveis durante as sessões de hemodiálise semanais, agregado a espera por estas, o que impede ou restringe suas atividades laborais e vida social. Há, além destes, outros fatores, como o fato de vivenciarem dificuldades na obtenção de transporte, problemas na recuperação, restrições alimentares, acúmulo de fadiga, ansiedade, deterioração nos relacionamentos familiares, se sentirem juntamente com seus familiares dependentes do centro de diálise, entre outras situações adversas (Gayomali et al., 2008; Sezer et al., 2003).

Vale salientar que a depressão é multifatorial, não sendo estes os únicos fatores para o desencadeamento desta patologia. Distintos aspectos como: variáveis genéticas, psicodinâmicas, desenvolvimentais, cognitivas e neurobiológicas, também podem ser predisposições à depressão (OMS, 2016).

Destarte, no que se referem as demais variáveis sociodemográficas dos cuidadores, não foram constadas associações significativas com a sintomatologia depressiva, como pode ser observado: $\operatorname{sexo} \chi^{2}(1,100)=0,01, p=0,60$; estado civil $\chi^{2}(1,100)=1,46, p=0,35$; religião $\chi^{2}(2,100)=1,81, p=0,91$; o grau de parentesco $\chi^{2}(2,100)=5,28, \mathrm{p}=0,07$, nem correlações, idade $r(1)=0,95, \mathrm{p}=$ $0,51)$; escolaridade $p(1)=-0,22, \mathrm{p}=0,11)$ e ocupação $p(1)=-0,12, \mathrm{p}=0,37)$.

\section{CONSIDERAÇÕES FINAIS}

Este artigo objetivou mensurar a sintomatologia depressiva em pacientes renais crônicos e seus familiares/cuidadores e relacioná-la as variáveis sociodemográficas. Para isso, utilizou-se da aplicabilidade da estatística descritiva e inferencial. Os resultados evidenciaram uma prevalência de $20 \%$ da sintomatologia depressiva entre os pacientes, classificados em $12 \%$ como sintomatologia depressiva leve, $6 \%$ moderada e $2 \%$ grave. Entre os familiares/cuidadores, foi detectada $46 \%$, distribuídos em $30 \%$ com sintomas leves, $10 \%$ moderados e $6 \%$ graves. Diante desses resultados, é possível inferir que os familiares/cuidadores são mais suscetíveis de serem acometidos pela sintomatologia da depressão.

Observou-se estatisticamente associação entre o estado civil dos pacientes, evidenciando menos casos entre os casados do que nos solteiros, sugerindo que para os primeiros ter o cuidado da companheira vai além dos cuidados físicos relacionados a doença, mas também o envolvimento afetivo. Por meio da análise do perfil sociodemográfico dos cuidadores, constatou-se predomínio do sexo feminino, em sua maioria esposas dos pacientes. Equivalente ao que é encontrado na literatura, notou-se também uma correlação positiva entre o tempo de acompanhamento e cuidados com o paciente e a presença de 
sintomas depressivos nos familiares. Com isto é possível inferir que quanto maior a durabilidade do cuidar, maior a predisposição na aquisição dos sintomas depressivos.

Dessa forma, espera-se que esses resultados possam subsidiar a implementação de programas de políticas públicas voltados para a assistência não apenas com o doente, mas também com o cuidador que por vezes são tratados como sub-prioritários, ou que são negligenciados. Portanto, sugere-se programas sociais com a finalidade de propiciar aos familiares/cuidadores maiores informações acerca de como cuidar da doença e do doente renal. Uma vez, que existe um descompasso entre o saber e o fazer que pode levar os cuidadores a ficarem predispostos a outros tipos de doenças tanto físicas quanto psicoafetivas.

Menciona-se algumas limitações desse estudo, a utilização de uma amostra não probabilística, comprometendo à possibilidade de generalização, assim como, a quantidade de participantes. Devido a pesquisa ter sido realizada somente em instituições hospitalares públicas, tal aspecto, inviabilizou observar se existem diferenças entre o contexto público e o privado. Destaca-se também, que a prevalência da depressão encontrada não se aplica a um diagnóstico, uma vez que se utilizou um instrumento para o rastreamento da depressão.

\section{REFERÊNCIAS}

Andrade, S. V., Sesso, R., \& Diniz, D. H. M. P. (2015). Desesperança, ideação suicida e depressão em pacientes renais crônicos em tratamento por hemodiálise ou transplante. Jornal Brasileiro de Nefrologia, 37(1), 55-63. doi: 10.5935/0101-2800.20150009

Arechabala, M. C., Catoni, M. I., Palma, E., \& Barrios, S. (2011). Depresión y autopercepción de la carga del cuidado em pacientes en hemodiálisis y sus cuidadores. Revista Panamericana de Salud Pública, 30(1), 74-9. doi: 10.1590/S1020-49892011000700011

Asti, T., Kara, M., Ipek, G., \& Erci, B. (2006). The experiences of loneliness, depression, and social support of Turkish patients with continuous ambulatory peritoneal dialysis and their caregivers. Journal of Clinical Nursing, 15(4), 490 - 97. doi: 10.1111/j.1365-2702.2006.01330.x

Besier, T., \& Goldbeck, L. (2011). Anxiety and depression in adolescents with CF and their caregivers. Journal of Cystic Fibrosis, 10(6), 435 - 442. doi: 10.1016/j.jcf.2011.06.012

Botega, N. J., Bio, M. R., Zomignani, M. A., Garcia Jr, C., \& Pereira, W. A. B. (1995). Transtornos do humor em enfermaria de clínica médica e validação de escala de medida (HAD) de ansiedade e depressão. Revista Saúde Pública, 29(5), 359-363. doi: 10.1590/S0034-89101995000500004 
Brasil. (2014). Ministério da Saúde. Secretaria de Atenção à Saúde. Departamento de Atenção Especializada e Temática. Diretrizes Clínicas para o Cuidado ao paciente com Doença Renal Crônica - DRC no Sistema Único de Saúde/ Ministério da Saúde. Brasília: Ministério da Saúde.

Cardoso, L., Vieira, M. V., Ricci, M. A. M., \& Mazza, R. S. (2012). Perspectivas atuais sobre a sobrecarga do cuidador em saúde mental. Revista da Escola de Enfermagem da Universidade de São Paulo, 46(2), 513-517. doi: http://dx.doi.org/10.1590/S0080-62342012000200033

Chilcot, J., Wellsted, D., \& Farrington, F. (2010). Illness representations are associated with fluid nonadherence among hemodialysis patients. Journal of Psychosomatic Research, 68(2), 203-212. doi: 10.1016/j.jpsychores.2009.08.010

Costa, F. G. (2013). Depressão e insuficiência renal crônica: representações sociais de pacientes em hemodiálise e cuidadores. Dissertação de Mestrado, Universidade Federal da Paraíba, João Pessoa. Recuperado de http://tede.biblioteca.ufpb.br:8080/bitstream/tede/6911/1/ArquivoTotal.pdf

Costa, F. G., Coutinho, M. P. L., Melo, J. R. F., \& Oliveira, M. X. (2014). Rastreamento da depressão no contexto da insuficiência renal crônica. Revista Temas em Psicologia,22(2), 445-455. doi: 10.9788/TP2014.2-14

Coutinho, M. P. L., \& Costa, F. G. (2015). Depressão e insuficiência renal crônica: uma análise psicossociológica. Psicologia \& Sociedade, 27(2), 448449. doi.org/10.1590/1807-03102015v27n2p449

Cuker, G. M., \& Fragnani, E. C. S. F. (2010). As dimensões psicológicas da doença renal crônica. Monografia de Conclusão de Curso de Psicologia, Universidade do Extremo Sul Catarinense, RS. Recuperado de http://www.bib.unesc.net/biblioteca/sumario/000044/0000440B.pdf

Dias, E. A. (2011). Sobrecarga vivenciada por familiares cuidadores de pacientes esquizofrênicos e sua relação com a depressão. Dissertação de mestrado, Faculdade de Ciências Médicas, Universidade Nova de Lisboa, Lisboa, Portugal. Recuperado de http://run.unl.pt/handle/10362/5932

Fernandes, J. (2009). Cuidar no domicílio e a sobrecarga do cuidador familiar. Dissertação de Mestrado, Faculdade de Medicina da Universidade de Lisboa. Recuperado de http://udcpplanaltomirandes.files.wordpress.com/2011/08/tese-demestrado-da-dra-jacinta.pdf

Ferreira, P. L., \& Anes, E. J. (2010). Medição da qualidade de vida de insuficientes renais crônicos: criação da versão portuguesa do KDQOL-SF. Revista Portuguesa de Saúde Pública, 28(1), 31-39. Recuperado de http://www.ensp.unl.pt/dispositivos-de-apoio/cdi/cdi/sector-depublicacoes/revista/2010/pdf/vol-1-2010/03-2010.pdf 
Fritz, C. O., Morris, P. E., \& Richler, J. J. (2012). Effect size estimates: current use, calculations, and interpretation. Journal of Experimental Psychology: General, 141(1), 2-18. DOI: 10.1037/a0024338

Gayomali, C., Sutherland, S., \& Finkelstein, F. O. (2008). The challenge for the caregiver of the patient with chronic kidney disease. Nephrol Dial Transplant., 23(12), 3749-3751. doi: 10.1093/ndt/gfn577

Kimmel, P. L., Cohen, S. D., \& Peterson, R. A. (2008). Depression in patients with chronic renal disease: where are we going? Journal of Renal Nutrition, 18(1), 99-103. doi: 10.1053/j.jrn.2007.10.020

Maciel, S. C., Melo, J. R. F., Dias, C. C. V., Silva, G. L. S., \& Gouveia, Y. B. (2014). Sintomas depressivos em familiares de dependentes químicos. Psicologia: Teoria e Prática, 16(2), 18-28. Recuperado em 22 de janeiro de 2016, de http://pepsic.bvsalud.org/scielo.php?script=sci_arttext\&pid= S1516-36872014000200002\&lng=pt\&tIng=pt.

Marcolino, J. A. M, Mathias, L. A. S. T, Piccinini Filho, L., Guaratini, A. A., Suzuki, F. M., \& Alli, L. A. C. (2007). Escala Hospitalar de Ansiedade e Depressão: Estudo da validade de critério e da confiabilidade com pacientes no préoperatório. Revista Brasileira de Anestesiologia, 57(1), 52-62. doi: 10.1590/S0034-70942007000100006

Marques, A. K. M. C., Landim, F. L. P., Collares, P. M., \& Mesquita, R. B. (2011). Apoio social na experiência do familiar cuidador. Ciência \& Saúde Coletiva, 16(Supl. 1), 945-955. doi: 10.1590/S1413-81232011000700026

Miyashita, M., Narita, Y., Sakamoto, A., Kawada, N., Akiyama, M., Kayama, M. ... Fukuhara, S. (2009). Care burden and depression in caregivers caring for patients with intractable neurological diseases at home in Japan. Journal of the Neurological Sciences, 276(2), 148-152. doi: 10.1016/j.jns.2008.09.022

Moura Junior, J. A., Souza, C. A. M., Oliveira, I. R., Miranda, R. O., Teles, C., \& Moura Neto, J. A. (2008). Risco de suicídio em pacientes em hemodiálise: evolução e mortalidade em três anos. Jornal Brasileiro de Psiquiatria, 57(1), 44-51. Recuperado de http://www.scielo.br/pdf/jbpsiq/v57n1/v57n1a09.pdf

Nifa, S., \& Rudnicki, T. (2010). Depressão em pacientes renais crônicos em tratamento de hemodiálise. Revista da Sociedade Brasileira de Psicologia Hospitalar, 13(1), 64-75. Recuperado de http://pepsic.bvsalud.org/pdf/rsbph/v13n1/v13n1a06.pdf

OMS (2016). World Health Organization. Depression. Disponível em: http://www.who.int/mediacentre/factsheets/fs369/en/.

Ramos, I. C., Queiroz, M. V. O., \& Jorge, M. S. B. (2008). Cuidado em situação de doença renal crônica: representações sociais elaboradas por adolescentes. Revista Brasileira de Enfermagem, 61(2), 193-200. doi: org/10.1590/S0034-71672008000200008 
Ribeiro, D. F., Marques, S. K. L., \& Ribeiro, R. C. H. (2009). Processo de cuidar do idoso em diálise peritoneal ambulatorial contínua no domicílio. Acta Paulista de Enfermagem, 22(6), 761-766. Recuperado de http://www.scielo.br/pdf/ape/v22n6/a06v22n6.pdf

Ribeiro, K. C. S., Coutinho, M. P. L., \& Nascimento, E. S. (2010). Representação social da depressão em uma Instituição de Ensino da Rede Pública. Psicologia Ciência e Profissão, 30(3), 448-463. doi: 10.1590/S141498932010000300002

Rudnicki, T. (2014). Doença renal crônica: vivência do paciente em tratamento de hemodiálise. Contextos Clínicos, 7(1), 105-116. doi: 10.4013/ctc.2014.71.10

Saeed, Z., Ahmad, A. M., Shakoor, A., Ghafoor, F., \& Kanwal, S. (2012). Depression in patients on hemodialysis and their caregivers. Saudi Journal of Kidney Diseases and Transplantation, 23(5), 946-52. doi: 10.4103/13192442.100869

Sansoni, J., Vellone, E., \& Piras, G. (2004). Anxiety and depression in community-dwelling, Italian Alzheimer's disease caregivers. International Journal of Nursing Practice, 10(2), 93-100. doi: 10.1111/j.1440172X.2003.00461.X

Schuster, J. T., Feldens, V. P., Iser, B. P. M., \& Ghislandi, G. M. (2015). Avaliação de sintomas depressivos em pacientes com insuficiência renal crônica submetidos à hemodiálise em Tubarão - Santa Catarina - Brasil. Revista da Associação Médica do Rio Grande do Sul, 59(1), 15-19.

Sezer, M. T., Eren, I., Ozcankaya, R., Civi, I., Erturk, J., \& Ozturk, M. (2003). Psychological Symptoms Are Greater in Caregivers of Patients on Hemodialysis Than Those of Peritoneal Dialysis. Hemodialysis International, 7(4), 332-337. doi: 10.1046/j.1492-7535.2003.00058.x

Smith, M. D., Hong, B. A., \& Robson, A. M. (1985). Diagnosis of depression in patients with end stage renal disease. The American Journal of Medicine, 79(2), 160-166.

Stasiak, C. E. S., Bazan, K. S., Kuss, R. S., Schuinski, A. F. M., \& Baroni, G. (2014). Prevalência de ansiedade e depressão e suas comorbidades em pacientes com doença renal crônica em hemodiálise e diálise peritoneal. Jornal Brasileiro de Nefrologia, 36(3), 325-331. doi: 10.5935/01012800.20140047

Vieira, C. P. B., Fialho, A. V. M., Freitas, C. H. A., \& Jorge, M. S. B. (2011). Práticas do cuidador informal do idoso no domicílio. Revista Brasileira de Enfermagem, 64(3), 570-579. Recuperado de http://www.scielo.br/pdf/reben/v64n3/v64n3a23.pdf 
Fabrycianne Gonçalves Costa é neuropsicóloga, mestre em Psicologia e doutoranda em Psicologia Social pela Universidade Federal da Paraíba, membro do Núcleo de pesquisa Aspectos Psicossociais de Prevenção e da Saúde Coletiva. E-mail: fabrycianne@gmail.com

Maria da Penha de Lima Coutinho é Doutora em Psicologia Clínica pela Universidade de São Paulo, pós-doutora pela Universidade Aberta de Lisboa, Portugal, professora do programa de Psicologia Social da Paraíba e coordenadora do Núcleo de pesquisa Aspectos Psicossociais de Prevenção e da Saúde Coletiva. E-mail: mplcoutinho@gmail.com

Recebido em: 19/07/2015 1 a revisão em: 30/01/2016 $2^{a}$ revisão em: 04/03/2016

Aceito em: $13 / 03 / 2016$ 